\title{
COMPREENSÃO DAS BASES TEÓRICAS DO PLANEJ AMENTO PARTICIPATIVO NO CURRÍCULO INTEGRADO DE UM CURSO DE ENFERMAGEM
} Maria Cristina M. Capel Laluna ${ }^{1}$
Clarice Aparecida Ferraz

Laluna MCMC, Ferraz CA. Compreensão das bases teóricas do planejamento participativo no currículo integrado de um curso de enfermagem. Rev Latino-am Enfermagem 2003 novembro-dezembro; 11(6):771-7.

O presente trabalho trata de estudo exploratório que buscou identificar as bases teóricas que sustentam o planejamento participativo pautado no referencial do Planejamento Estratégico Situacional, utilizadas no currículo integrado da graduação em enfermagem, para o desenvolvimento do desempenho do planejamento participativo. Os dados foram obtidos por meio da análise documental dos vinte e dois cadernos das Unidades Educacionais que compõem o currículo do Curso de Enfermagem da Faculdade de Medicina de Marília, objetivando reconhecer os recortes de conhecimentos que contribuíram para a formação dos conceitos de ator social, situação e produção social. Na análise dos resultados, foram reconhecidos a insuficiência das bases teóricas, o predomínio de objetivos de domínio cognitivo e a descrição dos desempenhos na forma de objetivos. Contudo, revelou haver tentativas na integração dos conhecimentos e suas sucessivas aproximações, no tocante ao planejamento participativo, buscando coerência com os princípios do currículo integrado.

DESCRITORES: educação em enfermagem; curriculo; planejamento participativo

\section{UNDERSTANDING THE THEORETICAL BASES OF PARTICIPATIVE}

\section{PLANNING IN THE INTEGRATED CURRICULUM OF A NURSING COURSE}

This paper deals with an exploratory study aimed at identifying the theoretical bases that sustain participative planning as described in the framework of Situational Strategic Planning, which are used in the integrated curriculum of undergraduate nursing education for the development of participative planning performance. Data were obtained through documentary analysis of twenty-two notebooks of the Educational Units that are part of the Marilia Medical School Nursing Course curriculum, with a view to recognizing those parts of knowledge that contributed to the formation of the social actor, situation and social production concepts. Result analysis recognized the lack of theoretical bases, as well as the predominance of knowledge aims and the description of performance as objectives. However, attempts were revealed to integrate knowledge and approximate them to participative planning, with a view to achieving coherence with the principles of the integrated curriculum.

DESCRIPTORS: education, nursing; curriculum; participative planning

\section{COMPRENSIÓN DE LAS BASES TEÓRICAS DE LA PLANIFICACIÓN PARTICIPATIVA EN EL CURRÍCULO INTEGRADO DEL CURSO DE ENFERMERÍA DE LA FACULTAD DE MEDICINA DE MARÍLIA}

El presente trabajo es un estudio exploratorio que buscó identificar las bases teóricas que sustentan la planificación participativa con base en el referencial de la Planificación Estratégica Situacional, utilizadas en el plan de estudios integrado del curso de pregrado de enfermería, para desarrollar el desempeño de la planificación participativa. Los datos se obtuvieron a través del análisis documental de los veintidós cuadernos de las Unidades Educaciónales que componen el plan de estudios del Curso de Enfermería de la Facultad de Medicina de Marília, con el objetivo de reconocer los recortes de conocimiento que contribuyeron a la constitución de los conceptos de actor social, situación y producción social. Al analizar los resultados, se reconocieron la insuficiencia de las bases teóricas, el predominio de objetivos de conocimiento y la descripción de desempeños en la forma de objetivos. Sin embargo, el análisis reveló la existencia de intentos de integración de los conocimientos y sus sucesivas aproximaciones con respecto a la planificación participativa, buscando coherencia con los principios del currículo integrado.

DESCRIPTORES: educación en enfermería; currículo; planificación participativa

\footnotetext{
${ }^{1}$ Mestre em Enfermagem pelo Programa de Pós-Graduação da Escola de Enfermagem de Ribeirão Preto, da Universidade de São Paulo, Área Enfermagem Fundamental, Professor da Faculdade de Medicina de Marília, e-mail: cricapel@famema.br; ${ }^{2}$ Professor Livre-Docente da Escola de Enfermagem de Ribeirão Preto, da Universidade de São Paulo, Centro Colaborador da OMS para o desenvolvimento da pesquisa em enfermagem, e-mail: erraz@eerp.usp.br
} 
A FORMAÇÃO DO PROFISSIONAL ENFERMEIRO

$\boldsymbol{A}$ enfermagem brasileira vem repensando seu modelo de formação mais intensamente na última década do século XX, considerando a situação atual do mercado de trabalho, as competências do pessoal de enfermagem de nível médio e superior, o processo de mobilização e participação nas entidades de classe e o seu papel na implementação do Sistema Único de Saúde-SUS.

O processo de reformulação do modelo de formação de enfermeiros deve expressar um sentido de transformação da realidade de saúde do país; dessa maneira, Cortella nos incita a pensar que "formar alguém que trabalhe com a realidade brasileira, é formá-lo com consciência dos determinantes sócio-históricos que a condicionam e, ao mesmo tempo, com uma sólida formação sociocientífica que permita enfrentar, competentemente, essa mesma realidade, não como um apelo à vontade apenas, mas à capacidade"(1)

Portanto, o projeto educativo que se supõe nessa direção deve prever práticas pedagógicas enriquecedoras do processo de ensino-aprendizagem, para que 0 estudante compreenda, criticamente e de forma contextualizada, a prática que exercerá profissionalmente, estabelecendo constante interação entre teoria e prática e entre ensino e trabalho ${ }^{(2)}$

Nessa direção, na década de 90, considerando o movimento mundial de reorganização do setor saúde, a Faculdade de Medicina de Marília - FAMEMA, - com o apoio técnico e financeiro da Fundação Kellogg, por meio do Projeto UNI - Uma Nova Iniciativa na formação dos profissionais da saúde, iniciou um redirecionamento do ensino de seus Cursos de Medicina e Enfermagem.

Desde 1993, o Projeto UNI-Marília vem sendo desenvolvido por meio de parceria entre a FAMEMA, Secretaria Municipal de Higiene e Saúde e Conselho Municipal de Saúde, respectivamente responsáveis pelos componentes - academia, serviço e comunidade - que, articulados, visam à construção de um sistema de saúde eficaz e eficiente, humanizado e estruturado segundo os princípios do SUS, com enfoque na vigilância em saúde e no processo de trabalho multiprofissional e interdisciplinar.

O Curso de Enfermagem, considerando as propostas do ideário UNI e, ainda, acompanhando as discussões sobre o ensino de Enfermagem, realizadas no Seminário Nacional de Diretrizes e Bases para Educação em Enfermagem- SENADEn ${ }^{(3)}$, reformulou seu currículo, culminando com a construção de um projeto político pedagógico que pretende formar enfermeiros competentes e comprometidos socialmente.

Esse projeto organiza-se na modalidade de currículo integrado e vem sendo defendido, por se tratar de uma opção pedagógica que propicia o ensino das ciências de forma integrada e interdisciplinar, criando oportunidades para os estudantes analisarem os problemas de saúde segundo o ponto de vista de várias disciplinas. Pode-se evidenciar, também, a importância da ação e da experiência na aprendizagem, tornando-a significativa, pois os incentivos para a continuidade da aprendizagem são provenientes do ambiente concreto, do qual emergem os conflitos que são capazes de suscitar o interesse e o desejo de intervir neles ${ }^{(4)}$.

Essa forma de educar possibilita a humanização do conhecimento na medida em que favorece diferentes visões da realidade, nas quais as pessoas aparecem como sujeitos da história, estimulando, assim, o desenvolvimento do compromisso, da participação ativa, responsável e crítica $^{(4)}$

O Curso de Enfermagem, com a construção do novo projeto político-pedagógico, pretende buscar a formação de um enfermeiro que seja capaz de trabalhar em equipe multiprofissional, com enfoque interdisciplinar; prestar cuidados de enfermagem; ter visão crítica da estrutura social; pautar suas ações pela ética profissional; gerenciar a assistência de enfermagem e os serviços de saúde; buscar constante capacitação e ser capaz de realizar pesquisa ${ }^{(5)}$.

A Pedagogia Crítico Social e a Metodologia da Problematização consistem nas bases teóricas e metodológicas que fundamentam o currículo do Curso de Enfermagem da FAMEMA. A Pedagogia Crítico Social atribui à educação "o papel de proporcionar aos alunos o domínio de conteúdos científicos, os métodos de estudo e habilidades e hábitos do raciocínio científico, de modo a irem formando consciência crítica face às realidades sociais e capacitando-se a assumir um conjunto de lutas sociais à sua condição de agente ativo de transformação da sociedade e a si próprios" ${ }^{\text {"(6) }}$.

A Metodologia da Problematização, considerando as rápidas transformações da realidade, encaminha para a necessidade de aumentar a capacidade do aluno como participante e agente da transformação social, desenvolvendo sua capacidade de "fazer perguntas relevantes em qualquer situação para entendê-las e resolvê- 
las adequadamente" ${ }^{\text {"(7) }}$. Portanto, requer um movimento de aprendizagem de ação-reflexão-ação.

Considerando esses fundamentos teóricos, o currículo integrado do Curso de Enfermagem visa articular os seus conteúdos e áreas de conhecimento por meio da interdisciplinaridade; para tanto, os conteúdos selecionados foram distribuídos em 22 Unidades Educacionais - UE's que compõem a grade curricular. Para cada UE, desempenhos foram concebidos objetivando remeter o estudante à competência profissional. Entendemos por desempenho as ações desenvolvidas no processo ensino-aprendizagem, relativas ao trabalho do enfermeiro.

O desempenho permite reconhecer o que o estudante faz com o objeto apreendido, podendo vir a ser "a expressão concreta dos recursos que o indivíduo articula e mobiliza no enfrentamento de situações", e, assim, possibilita reconhecer a utilização que ele faz daquilo que sabe, por isso é tomado como objeto de avaliação ${ }^{(8)}$.

Considerando o pretendido no referido projeto político pedagógico e as reflexões produzidas durante os momentos de capacitação docente acerca da formação e prática do enfermeiro, o corpo docente das Disciplinas de Administração Aplicada à Enfermagem e Saúde Coletiva selecionou e adotou o referencial teórico e metodológico do Planejamento Estratégico Situacional - PES, desenvolvido por Carlos Matus.

No entender dos docentes, o referencial do PES colabora na construção da competência gerencial do enfermeiro, por constituir-se em um planejamento integrado e participativo, o que contribui para a organização dos serviços de saúde e para o enfrentamento de conflitos e problemas institucionais.

Isso posto, declaramos que desenvolvemos nossa dissertação de mestrado focando o ensino-aprendizagem no referido currículo integrado, priorizando dois objetivos: a identificação dos recortes de conhecimento desenvolvidos nas UE's, para a formação das bases teóricas do PES, e a análise da aplicação desses recortes pelos estudantes, na construção do desempenho para o planejamento participativo.

Esse artigo que ora apresentamos é um excerto do trabalho de mestrado, para o qual priorizamos a primeira fase da pesquisa, que teve, como objetivo, identificar os recortes de conhecimento desenvolvidos nas UE's do projeto pedagógico do Curso de Enfermagem da FAMEMA, que contribuíram para a formação das bases teóricas do
PES, proposto por Carlos Matus.

\section{BASES TEÓRICAS DO PLANEJAMENTO}

O referencial do PES, proposto por Carlos Matus, é uma ferramenta potente para transformar a realidade, visto que proporciona um aumento na capacidade de direção, gerência, administração e controle do sistema social. Por ser um método de processar problemas, tem grande aplicabilidade na gestão em saúde, uma vez que o trabalho, nessa área, trata de problemas complexos e não estruturados ${ }^{(9)}$.

O planejamento é o cálculo que precede e preside a ação, possibilitando ao homem conduzir seu futuro e impor-se ao contexto por meio da razão. Os problemas dos homens não são resolvidos de forma determinista, por isso o cálculo deve ser situacional e sistemático, articulando diversas extensões de tempo e várias dimensões da realidade. O planejamento, então, está determinado pela movimentação social e pela dinâmica da produção e reprodução da sociedade ${ }^{(10)}$.

A complexidade dos problemas decorre da compreensão de que a produção social é gerada em um sistema humano que tem, como características, a historicidade, a complexidade, a indefinição e a incerteza. Portanto, não se aceita uma única explicação ou a predição do futuro, exigindo-se uma solução situacional, discutível e dependente dos interesses e da posição dos atores envolvidos ${ }^{(10)}$.

As organizações complexas, como são os serviços de saúde, produzem relações de conflito e de articulação entre as forças sociais, o que, ao nosso ver, requer uma tecnologia de planejamento que consiga propiciar o enfrentamento dos problemas e a obtenção de soluções criativas.

Passamos a descrever as bases teóricas do PES proposto por Carlos Matus, as quais se delineiam em três conceitos fundamentais: ator social, situação e produção social.

Conceito de ator social

O conceito de homem estrutura-se como o de um ator social. "É uma personalidade, uma organização, ou um agrupamento humano, que, de certa forma, estável ou transitória, tem capacidade de acumular força e 
desenvolver interesse, produzindo fatos na situação"(10).

O homem de ação, implícito no conceito de ator social, é criativo e inserido em uma realidade, em interação com os outros, o que the possibilita ser estratégico na construção de projetos e na intervenção.

Nesse enfoque, a ação é entendida como sendo situacional, portanto passível de várias interpretações, as quais dependem da auto-referência dos atores envolvidos e "somente é concreta numa situação concreta e aí se define com distintos significados para os diversos atores sociais" $^{\text {(10) }}$

Conceito de situação

Situação remete ao espaço social em que se encontram os atores e a ação, criando possibilidades para a "explicação da realidade, elaborada por uma força social em função da sua ação e da sua luta com outras forças sociais. Ela refere-se ao ator da explicação, seus oponentes e aliados, suas ações, às ações de seus oponentes e aliados, assim como à realidade social, construída no processo social. Essa realidade apresentase como dada num certo momento da formação social, mas é produto da luta constante por manter, reproduzir e transformar o sistema"(10).

Esse conceito nos leva a considerar que a realidade é explicada por quem está dentro dela, ocupando um determinado espaço, em um determinado tempo, estabelecendo relações com ela. E, assim, sua ação tem sentido porque está na situação. Tal conceito é a chave para entender o outro e assimilar o seu ponto de vista. Portanto, situação é um recorte da realidade, percebido pelo ator social.

\section{Conceito de produção social}

A Teoria da produção social reporta-se à forma de produzir e de reproduzir de uma sociedade historicamente situada. Portanto, trata-se de uma construção de atores que estão expostos a certas condições e limitações.

Compreender a sociedade como um sistema produtivo significa considerar as várias dimensões da realidade onde são desenvolvidos fatos de natureza política, econômica e social. Significa, ainda, levar em conta que esse é um sistema conflitivo e criativo.

Produção social, então, pode ser entendida como um sistema em que toda a realização efetuada por um ator social utiliza-se de recursos econômicos, de poder e de aplicações de conhecimentos. Dessa forma, "toda a produção social reverte ao ator-produtor como acumulação social (ou desacumulação) de novos recursos econômicos, de poder, reafirmação, questionamento, ou surgimento de vários valores e acumulação de novos conhecimentos"(10).

\section{PERCURSO METODOLÓGICO}

O diferencial de um currículo integrado está na seleção e articulação de conteúdos interdependentes, na integração da teoria e prática, colocando importância na experiência de aprendizagem, em ambientes concretos de prática profissional. Considerando tais princípios, a primeira fase da pesquisa foi estruturada para identificar os conteúdos selecionados para o processo ensinoaprendizagem do PES, bem como a sua articulação no conjunto das UE's do currículo integrado do Curso de Enfermagem da FAMEMA.

O procedimento adotado foi a análise de documentos do Curso de Enfermagem, especificamente os cadernos de registros das vinte e duas UE's da grade curricular. As informações foram coletadas no período de julho a outubro de 2001, após aprovação do Comitê de Ética em Pesquisa da FAMEMA.

Primeiramente, realizamos leitura isolada de cada UE, estabelecendo relações entre os objetivos propostos, os conteúdos e os desempenhos esperados. Posteriormente, procedemos leitura conjunta das UE's, especificamente das seqüências de atividades, buscando a rede explicativa dos conceitos do PES, delimitando os recortes de conhecimento sobre ator social, situação e produção social. Para efetuar a análise dos objetivos, utilizamos a taxionomia de Bloomm, Krathwhl e Harrow ${ }^{(11)}$ e, para a análise dos desempenhos, utilizamos a compreensão de que eles são construídos por atributos cognitivos, atitudinais e habilidades psicomotoras ${ }^{(12)}$.

\section{ANÁLISE DOS RESULTADOS}

$\mathrm{Na}$ análise os objetivos, os recortes de conhecimento e os desempenhos esperados para o PES foram agrupados de forma sistemática por UE, a fim de evidenciar as contribuições de cada uma em particular. A seguir, apresentamos os resultados segundo análise dos conceitos que compõem a base do PES. 
Conceito de ator social

Das 22 UE's, 19 contribuíram com recortes de conhecimento para a compreensão do conceito de ator social, pois estiveram voltadas ao entendimento do homem como ser social, histórico, com diferenças biopsicossocioculturais. Interpretamos que a compreensão de ser social é desenvolvida ao abordá-lo como parte de um coletivo que, por meio do trabalho, produz e se reproduz, caracterizando, assim, uma possibilidade de ação reflexiva e intencional.

A identificação dos recortes relacionados ao trabalho em grupo e em equipe, bem como os da enfermagem como força social reforça a idéia de que o ator social é um ser coletivo, com interesses distintos e reconhece que a força do coletivo pode mudar o curso do processo social.

Nota-se que os recortes relacionados ao sujeito particular ficaram restritos à determinação biológica, sendo pouco expressivos no que se refere ao plano do desejo e do interesse; contudo, são de extremo valor para a compreensão das ações humanas.

Um recorte de conhecimento pouco explorado, porém importante para o conceito de ator social em ação, refere-se ao processo de comunicação específico dos atos da fala e do processo de negociação. Não houve clareza quanto aos conhecimentos relacionados às estruturas de poder na descrição do recorte de conhecimento sobre relacionamento interpessoal, em grupo e em equipe.

Pudemos, ainda, perceber uma discrepância entre as UE's, com respeito aos recortes de conhecimentos relativos às dimensões biopsicossocioculturais. Observamos o predomínio dos referentes à dimensão sociocultural nas UE's do primeiro ano do curso, sendo pouco expressivos nas subseqüentes. Os correspondentes à dimensão biológica e, em menor intensidade, à psicológica foram identificados nas demais unidades, reproduzindo a fragmentação do homem, característica do ensino disciplinar.

Ao relacionarmos os desempenhos esperados, notamos o predomínio da ação de domínio cognitivo, excetuando o referente ao trabalho em grupo. Os objetivos relativos a atitudes e habilidades foram pouco expressivos, o que se confirmou quando reconhecemos uma maior incidência dos objetivos de domínio cognitivo, em que os comportamentos esperados foram os relativos ao conhecimento e à compreensão, com alguns indicativos pertinentes à aplicação. Isso denota que os estudantes não demonstraram a aplicação do conceito de ator social à prática.

A pequena aparição de objetivos e desempenhos atitudinais e de ação e a forma fragmentada do desenvolvimento dos recortes de conhecimento chamaramnos a atenção por demonstrarem a insuficiência do desenvolvimento do conceito de ator social no decorrer das UE's.

Conceito de situação

Os recortes de conhecimento trabalhados no decorrer das 22 UE's referiam-se a diferentes formas de conceber o mundo, as quais determinam as ideologias que sustentam a caracterização de uma determinada situação. Identificamos os relacionados ao diagnóstico técnico das condições de saúde da população, diferenciando os problemas bem estruturados daqueles quase estruturados, considerando a determinação do processo saúde-doença. Encontramos, ainda, os que diziam respeito à caracterização dos serviços de saúde, das práticas de saúde desenvolvidas pelos profissionais e pela população, bem como os referentes às políticas de saúde que sustentam o modelo de assistência proposto pelo SUS.

Entretanto, não evidenciamos, no conjunto das UE's, mais especificamente nos desempenhos e nos atributos atitudinais, a auto-referência do ator, identificandose com a leitura da realidade, com o contexto e as diferenças de interesses, os quais devem ser considerados na apreciação situacional. Sendo assim, pareceu-nos que a explicação técnica e objetiva ainda esteve preponderante na explicação da situação.

Notamos, também, no conjunto das UE's, o predomínio dos objetivos e dos desempenhos na área do domínio cognitivo, privilegiando os de conhecimento e compreensão. Visualizamos, todavia, uma pequena aparição no de aplicação, seguida pelos domínios afetivo e psicomotor. Novamente, reconhecemos a insuficiência no desenvolvimento do conceito de situação ao nos depararmos com a descrição de desempenhos, em sua maioria, em forma de objetivos.

Conceito de produção social

Os recortes de conhecimento acerca da produção 
social são referentes à forma de produzir e organizar as ações de saúde para atender às necessidades sociais advindas do modo de produção capitalista. Em sete UE's, estiveram presentes os recortes pertinentes à divisão do trabalho, suas relações com o homem e as conseqüências para ele quando há separação do trabalho manual e do intelectual, conforme demonstrados nas UE's do primeiro ano do curso. Com relação à produção de serviços, correspondendo ao plano dos fluxos, identifica-se a produção de ações de cuidado à saúde do indivíduo, da família e da comunidade, interligadas às políticas e, portanto, inseridas no plano das regras. Não fica explicitada, porém, a natureza das ações produzidas no cotidiano e as oferecidas pelo sistema social.

Encontramos em uma UE do terceiro ano, o recorte de conhecimento referente a dados de produção do serviço hospitalar, porém sem explicitação das suas relações com o plano dos fluxos, das acumulações e das regras.

Consideramos insuficiente o desenvolvimento desse conceito, uma vez que, na teoria da produção social, a produção econômica, conforme Matus, é apenas um dos recursos escassos que o homem enfrenta, e a realidade não pode ser explicada somente por essa perspectiva. A produção realizada pelo ator social também deve ser ampliada por recursos de poder, de aplicação de valores e de conhecimentos, de comunicação e de organização.

Os resultados da primeira fase da investigação revelaram haver tentativas de articulação entre os conhecimentos e suas sucessivas aproximações no tocante ao planejamento participativo, buscando coerência com os princípios do currículo integrado.

Contudo, pudemos perceber o descompasso entre objetivos, recortes de conhecimento e desempenhos, bem como a prevalência de objetivos e desempenhos relacionados ao domínio cognitivo, mais especificamente a conhecimento e compreensão.

Constatamos que, com a construção dos desempenhos no formato de objetivos, a certificação dos desempenhos não deve ter ocorrido, mostrando que o currículo ainda apresenta forte característica de um currículo por objetivos e não por competências e desempenhos, conforme deveria constar no currículo integrado. Esse fato reflete a inexperiência do corpo docente com relação ao processo didático, em seus elementos constitutivos.

A nosso ver, os recortes de conhecimento desenvolvidos favoreceram o desenvolvimento parcial dos conceitos de ator social, situação e produção social. A articulação entre as disciplinas está em fase inicial e o caminho a ser percorrido até a interdisciplinaridade ainda está em processo de construção, conforme percebido pela desarticulação dos conceitos trabalhados no decorrer das UE's.

\section{SÍNTESE PROVISÓRIA}

A possibilidade de (re)olhar os cadernos das UE's para proceder à análise documental permitiu reconhecer os objetivos, os conhecimentos e os desempenhos desenvolvidos que contribuíram para a compreensão das bases teóricas do PES.

As UE's que desenvolveram os recortes de conhecimentos necessários à formação do conceito de ator social, situação e produção social direcionaram a maioria dos desempenhos esperados dos estudantes para o desenvolvimento do domínio cognitivo, especificamente aos comportamentos de conhecer e compreender.

O conhecer representa a memorização, e o compreender traz a idéia de entendimento dos conteúdos transmitidos, ambos esperados na fase inicial da aprendizagem, segundo Bloom, não garantindo que serão aplicados à prática ${ }^{(11)}$.

Reconhecemos que os recortes de conhecimento que sustentam o planejamento participativo não foram desenvolvidos na sua totalidade, considerando que as UE's que desenvolveram os conceitos de ator social, situação e produção social foram construídas de forma fragmentada, com pouca articulação entre si, o que reafirma que seus conteúdos foram trabalhados de forma predominantemente disciplinar.

A rediscussão dos conteúdos relevantes e necessários à transformação da realidade de saúde e a aproximação com o conhecimento didático pedagógico, em relação à construção de objetivos educacionais, desempenhos, competências, interdisciplinaridade e avaliação podem diminuir a distância entre o currículo idealizado e o praticado.

Diante das análises evidenciadas, destacamos a importância de se estudar a construção das seqüências de atividades, considerando-se a metodologia da problematização, os esquemas mentais de assimilação representação, relação e ação no desenvolvimento dos conceitos-chave. 
A avaliação é outro tema relevante para investigação em que destacamos a análise de processos avaliativos que acompanhem a aplicação combinada dos atributos em situação prática.

Tais estudos poderiam trazer relevantes contribuições e propiciar ajustes importantes para o curso de Enfermagem da FAMEMA, bem como favorecer outras mudanças curriculares em andamento, uma vez que constatamos que apenas a verificação dos conteúdos desenvolvidos, objetivos e desempenhos não foi suficiente para analisar os nós críticos do processo ensinoaprendizagem.

\section{REFERÊNCIAS BIBLIOGRÁFICAS}

1. Cortella MS. Determinantes do perfil e competência profissional. Anais do Seminário da região sudeste sobre perfil e competência do enfermeiro e o currículo mínimo para a graduação em enfermagem; 1988, São Paulo, SP. São Paulo: Escola Paulista de Medicina; 1988. p. 34-7.

2. Bagnato MHS. Fazendo a travessia: em pauta a formação dos profissionais da área da saúde. In: Bagnato MHS, Cocco MIM, Sordi MRL. organizadoras. Educação, saúde e trabalho: antigos problemas, novos contextos, outros olhares. Campinas (SP): Alínea; 1999. p. 9-24.

3. Seminário Nacional de Diretrizes para Educação em Enfermagem no Brasil, 4, 2000. Relatório final. Fortaleza (CE): Associação Brasileira de Enfermagem - Seção Ceará; 2000. 4. Santomé JT. Globalização e interdisciplinaridade: o currículo integrado. Porto Alegre (RS): Artmed; 1998.

5. Chirelli $M Q$, coordenadora. Introdução ao Curso de Enfermagem. Marília (SP): Curso de Enfermagem da Faculdade de Medicina de Marília; 1999.

6. Libâneo J. Didática. São Paulo (SP): Cortez; 1999.

7. Dias Bordenave JE. Alguns fatores pedagógicos. In: Ministério da Saúde (BR). Secretaria Geral. Secretaria de Modernização Administrativa e Recursos Humanos. Capacitação Pedagógica para instrutor/supervisor- área da saúde. Brasília (DF): Ministério da Saúde; 1989.

8. Ramos MN. Qualificação, competências e certificação: visão educacional. In: Ministério da Saúde (BR). Secretaria de Gestão de Investimentos em Saúde. Projeto de Profissionalização dos Trabalhadores da Área de Enfermagem - PROFAE. Humanizar cuidados de saúde: uma questão de competência. Brasília (DF):Ministério da Saúde; 2001. p. 17-26.

9. Fekete MC. Roteiro de apoio: bases conceituais e metodológicas do planejamento em saúde. In: Santana JP, coordenador. Desenvolvimento gerencial de unidades básicas de saúde do distrito sanitário-Projeto Gerus. Brasília (DF): Ministério da Saúde; 1995. p. 81-5.

10. Matus C. Política, planejamento \& governo. Brasília (DF): IPEA; 1993.

11. Blomm BS, Engelhart MD, Edward JF, Hill WH, Kratwohl DR. Taxionomia de objetivos educacionais: domínio cognitivo. Porto Alegre (RS): Globo; 1974.

12. Depresbiteris L. Certificação de competências: a necessidade de avançar numa perspectiva formativa. In: Ministério da Saúde (BR). Secretaria de Gestão de Investimentos em Saúde Projeto de Profissionalização dos Trabalhadores da Área de Enfermagem - PROFAE. Humanizar cuidados de saúde: uma questão de competência. Brasília (DF):Ministério da Saúde; 2001. p. 27-38. 